This item was submitted to Loughborough's Research Repository by the author.

Items in Figshare are protected by copyright, with all rights reserved, unless otherwise indicated.

\title{
A holistic, learning-centred approach to building evaluation capacity in development organizations
}

PLEASE CITE THE PUBLISHED VERSION

http://dx.doi.org/10.1177/1356389015590219

PUBLISHER

SAGE ( ) The Author(s)

VERSION

AM (Accepted Manuscript)

\section{PUBLISHER STATEMENT}

This work is made available according to the conditions of the Creative Commons Attribution-NonCommercialNoDerivatives 4.0 International (CC BY-NC-ND 4.0) licence. Full details of this licence are available at: https://creativecommons.org/licenses/by-nc-nd/4.0/

\section{LICENCE}

CC BY-NC-ND 4.0

\section{REPOSITORY RECORD}

Lennie, June, Jo Tacchi, Michael Wilmore, and Bikash Koirala. 2019. "A Holistic, Learning-centred Approach to Building Evaluation Capacity in Development Organizations". figshare. https://hdl.handle.net/2134/23582. 


\section{A holistic, learning-centred approach to building evaluation}

\section{capacity in development organisations}

June Lennie, Jo Tacchi, Michael Wilmore and Bikash Koirala

Paper submitted to Evaluation on 22-10-2014 then revised on 17-04-2015

\section{Introduction}

Growing pressures from donors to demonstrate accountability and value for money through impact evaluation, plus increased emphasis on 'development partnerships, local ownership and good governance' (Dabelstein, 2003: 367) have led to increased interest in evaluation capacity development (ECD; also known as evaluation capacity building) amongst development organisations. ${ }^{1}$ This interest in ECD has been driven by a number of factors, especially growing appreciation of the value of participatory, systems and complexity-based approaches for evaluating complex development interventions (Armytage, 2011; Morgan, 2013; Patton, 2010). The use of external evaluators to undertake evaluations of development initiatives is also frequently questioned given that they often lack a good understanding of the local context and are unable to 'adequately capture what is going on' (Naccarella et al., 2007: 231). Sonnichsen (1999: 56) suggests that a benefit of using internal evaluators is that they 
'have a long-term commitment and can act in the capacity of change agents, increasing organisational performance'. In addition, longitudinal research by Robinson and Cousins (2004) has clearly demonstrated the positive effect of internal participatory evaluation on organisational learning. Related research has highlighted the positive role of critical reflection and evaluation in developing learning organisations, which enables them to effectively respond and adapt in complex and rapidly changing contexts, such as those in developing countries (Behrens and Kelly, 2008; Hay, 2010; Pearson, 2011).

However, improving and sustaining evaluation capacities often present particularly difficult challenges for organisations based in developing countries, because they are often time, capacity and resource poor (Lennie and Tacchi, 2013). Efforts to introduce ECD into organisations' work may be hindered by the same factors that development organisations actually seek to address in their work, such as the vulnerability and unpredictable nature of institutional systems in developing societies, high levels of poverty, and lack of 'resources, opportunities, and exposure to new ideas' (Ofir and Kumar, 2013: 14). There are also significant costs associated with effective use of ECD approaches (Cousins et al., 2013), because they may require new 'people skills' (Patton, 2010: 49). While various ECD approaches and models have been developed, 
they tend to neglect the significant challenges and issues experienced by organisations in development contexts.

Consequently, this paper addresses an urgent need to improve our understanding of the barriers that stand in the way of effective ECD and to identify approaches that can overcome these without undermining the essential aims of ECD. We argue that ECD requires long-term commitment to capacity development that may be at odds with the focus on one-off workshops and short-term capacity development of individual staff that tend to dominate in the international development field due to the exigencies of project-based financing. Kuzim (2010: 240) points out that 'although training is important in the [evaluation capacity building] ECB process, it is not sufficient for building a sustainable evaluation capacity in organizations'. This is supported by a recent international workshop that identified the 'superficial' results of such approaches (Bayley et al., 2012: 5). Stern et al. (2012) suggest that new strategies are needed to enhance the capacity of development agencies and evaluators to support the uptake of a broader range of designs and methods for impact evaluation in international development. 
Our argument is based on evidence drawn from a project, Assessing Communication for Social Change (AC4SC), undertaken in collaboration with Equal Access Nepal (EAN), an NGO that produces radio programs focussed on social change. We explain the emergence of the holistic, learning-centred approach to ECD in this project before outlining the key features of this approach and how they were applied in this case. The project drew on approaches from a number of related fields and combined them to create an approach to ECD that is particularly appropriate for development organisations working in fields like communication for development (C4D), that often struggle to produce strong evidential bases for impact evaluation due to the complexity of causal connections between interventions and individual or community behaviours (Lennie and Tacchi, 2013). We also outline some of the challenges and issues that inevitably arise when using this approach in developing countries like Nepal that have struggled to improve standards of living against a backdrop of chronic political instability and civil war. Drawing on learnings from AC4SC and related research, we identify essential principles for ECD and strategies for the sustainable implementation of this approach to evaluation. 


\section{Evaluation capacity development: definitions and issues}

Most definitions of ECD emphasise that it 'is a complex phenomenon involving issues of individual learning, organizational change, sustained change and program processes and outcomes' (Labin et al. 2012: 328). Definitions also emphasise that it involves providing staff with the skills to conduct rigorous evaluations, and doing this in a way that both acknowledges the local context and 'ensures that such evaluations become part of routine practice' (Naccarella et al. 2007: 232). This requires ECD to 'foster a culture of organizational support for evaluation activities, which includes an appropriate learning environment and a sufficient level of resources' (Naccarella et al., 2007: 232). Most writers also agree that ECD is about 'increasing the sustainability of professional evaluation practice' (Preskill, 2010: 224). Ba Tall (2009: 123) suggests that $E C D$ is a continuing long-term process of learning and change management. A key lesson from international ECD experience is that 'building an effective capacity for monitoring and evaluation is neither quick nor easy' and there is a need for 'steady and sustained support by international donors' (Schiavo-Campo, 2005: 13).

Preskill and Boyle (2008) have developed a useful, comprehensive conceptual model of ECD. Taking a systems approach, this model 'illustrates and describes a set of factors that may influence the initiation, design, implementation, and impact that $\mathrm{ECB}$ 
activities and processes have on sustainable evaluation practice' (Preskill and Boyle, 2008: 444). Based on the concepts in their model, they provide the following detailed definition:

$E C B$ involves the design and implementation of teaching and learning strategies to help individuals, groups, and organizations, learn about what constitutes effective, useful, and professional evaluation practice. The ultimate goal of ECB is sustainable evaluation practice - where members continuously ask questions that matter, collect, analyze, and interpret data, and use evaluation findings for decision-making and action. For evaluation practice to be sustained, participants must be provided with leadership support, incentives, resources, and opportunities to transfer their learning about evaluation to their everyday work. Sustainable evaluation practice also requires the development of systems, processes, policies, and plans that help embed evaluation work into the way the organization accomplishes its mission and strategic goals (Preskill and Boyle, 2008: 444)

Preskill and Boyle's model has some basic similarities to the ECD approach outlined in this paper but does not take the particular challenges and issues of the development context into account. The focus of this model on embedding evaluation into the 
everyday practices of an organisation and using evaluation to help guide and support decision making and action has been labelled 'mainstreaming evaluation' (Fitzpatrick et al., 2012: 236). This is a key aspect of the holistic approach to ECD that we advocate and is particularly important to development organisations that must continually adjust their practice in response to constantly changing goals and conditions. Lennie and Tacchi (2013: 94) point out that developing evaluation capacity can be seen as 'part of the process of institutionalizing evaluation and creating an evaluation culture within development agencies and their government and NGO implementing partners'. They see this process as vital to widening appreciation of the value of C4D in reaching development goals.

The ECD approach detailed in this paper draws on Lennie and Tacchi's (2011) research to develop a UN Inter-agency Resource Pack on Research, Monitoring and Evaluation in C4D. This included an in-depth review of literature on evaluation and ECD in the development and C4D fields and consultations with an international expert panel and specialists from seven United Nations (UN) agencies and other bodies. This research identified a lack of evaluation capacity at all levels, especially in approaches that were considered more effective for evaluating C4D, and lack of opportunities for ongoing capacity development, training and support. The research also identified many 
challenges and issues for evaluating C4D that apply to the evaluation of complex development initiatives more broadly.

A comprehensive, overarching framework for evaluating C4D has been developed, which includes a significant focus on evaluation capacity development (Lennie and Tacchi, 2013; Tacchi and Lennie, 2014). The framework responds to the main challenges and issues identified in AC4SC and in the research for the UN Inter-agency Resource Pack. It comprises seven inter-related components: participatory, holistic, complex, critical, emergent, realistic and learning-based, which are each underpinned by a set of principles (see Figure 1).

Insert about here Figure 1: Key components and concepts in the framework for evaluating C4D (from Lennie and Tacchi, 2013: 143)

\section{Introducing our case study of the AC4SC project}

While ECD in developed countries is usually focussed at organisational and individual levels, Carter (2013: 3) notes that lessons learned on the process of ECD in developing countries highlight the importance of working at three interdependent levels of capacity: 'the enabling environment, the institutional framework and the individual'. 
We draw on evidence related to each of these levels derived from a case-study, Assessing Communication for Social Change (AC4SC) that is particularly valuable because it is based on a seven-year longitudinal study of the implementation of a participatory, holistic approach to ECD. Originally planned to last for four years from 2007 to 2011, the AC4SC project provided significant learnings about ECD in complex and challenging development contexts, especially through opportunities to do further evaluation of on-going impact after the official completion of the funded project. AC4SC developed, implemented and evaluated a participatory methodology for assessing the social and behavioural change impacts of radio programs made by Equal Access Nepal (EAN). This action research project was a collaboration between researchers from Queensland University of Technology and the University of Adelaide in Australia, Equal Access International, based in San Francisco, USA, EAN, local stakeholders, and a network of trained community researchers (CRs) in five diverse districts in Nepal. As far as possible, the project attempted to implement a holistic, learning-based approach to ECD within EAN and to find ways to make the participatory monitoring and evaluation (PM\&E) systems that were established sustainable.

EAN staff had previously been trained in ethnographic action research (EAR) in 2005 and 2006. This methodology appealed to EAN because it was participatory, focussed on improving information and communication technology (ICT) for development 
initiatives, and has a key aim of developing a research culture through which knowledge and reflection becomes integral to a communication initiative's ongoing development (Tacchi, forthcoming; Tacchi et al., 2007). While EAR was considered useful, EAN found it difficult to apply it consistently or systematically. The initial idea behind AC4SC was therefore to develop EAR into a methodology that was embedded in EAN. Previous models had relied on one or two EAR researchers working within and attempting to influence an organisation. However, the aim of AC4SC was to transform the organisation by establishing stronger and more effective M\&E systems and processes within it and to develop better M\&E skills and capacities, including collaboratively planning evaluations and managing and analysing qualitative data.

The earlier use of EAR provided some understanding of EAN's research and evaluation interests and needs and helped to develop good relationships with staff at all levels. The AC4SC project involved working closely with a range of EAN staff, following a participatory action research (PAR) approach, to develop systems and processes to assess the impacts of two community radio programs. With the collaboration and support of the Australian research team, EAN's M\&E team developed research plans, built and trained a network of CRs, collected and organised data from sites across Nepal, developed systems of coding and data analysis, and reported regularly to content teams (producers) and management. 
We undertook an ongoing meta-evaluation of the project that assessed the effectiveness of this approach (Lennie et al., 2012). Our case study draws on the findings from the meta-evaluation as well as:

- A detailed analysis of in-depth interviews conducted by June Lennie in 2009 with nine EAN staff comprising two senior management staff, three M\&E staff and four content production or program management staff.

- A report on follow up research on EAN's M\&E systems and the CR network by Michael Wilmore and Bikash Koirala in January 2012, ten months after the project ended.

- Notes from a further follow up meeting at EAN's office in Kathmandu that June Lennie and Jo Tacchi took part in with 14 staff in February 2013, at which an update on AC4SC-related activities and impacts was provided.

- Recent information about M\&E systems and practices in EAN and the long-term impacts of the project provided by Bikash Koirala, an M\&E Officer at EAN who played a significant role in AC4SC and the development of the PM\&E toolkit that emerged from the project. 


\section{Barriers to successful implementation of evaluation capacity development}

As we have indicated, there are many tensions, challenges and issues in building evaluation capacities in development contexts, and in using participatory and holistic approaches to ECD, including contextual and cultural challenges, organisational culture and power issues, staff turnover, negative attitudes to $M \& E$, resistance to change and lack of time and resources, including funding for ECD activities (Lennie and Tacchi, 2013; Lennie and Tacchi, 2014; Tacchi et al., 2013). We discuss some of these challenges and issues below.

Contextual and cultural issues can hinder the effective implementation of a holistic approach to ECD within time, capacity and resource-poor organisations in developing countries. A key issue is that development organisations often face pressure to meet the upward accountability needs of donors rather than the learning needs of organisations and communities (Lennie and Tacchi, 2014). Many C4D initiatives are not well equipped to deal with the challenges and complexities of evaluating C4D, given the various constraints and difficult contextual tensions and challenges that they face.

An important learning from Pearson's (2011) capacity building efforts with a Cambodian NGO was that only an understanding of local culture and the recent history 
of the Cambodian people could help to explain the resistance of staff to new ways of thinking and learning. We faced similar challenges during AC4SC which affected the success of our ECD activities. They included language and communication problems and other factors related to the complexity of the social and cultural context, not the least of which was the aftermath of a decade long civil war in Nepal. This raises questions about the influence of cultural contexts on ECD because participant's expectations of their own and each other's roles vary depending on their previous experiences and backgrounds. For example, the relative informality of Australian academic institutions contrasts greatly to the expectations of deference that typically characterise the relationships of Nepali students to their teachers (Tacchi et al., 2013). In addition, due to the lack of awareness among some community groups about M\&E and its benefits, they were sometime reluctant to take part in AC4SC-related activities. Achieving effective and sustainable ECD requires taking into account the organisational culture, dynamics and wider context, and issues of gender, power and knowledge. Organisations form networks of people with different agendas and interests and varying levels of power, status, authority, experience and expertise (Cracknell, 2000). The degree of conflict and cooperation among these groups has an impact on ECD and evaluation activities. Gender and power relations are likely to affect ECD processes, 
especially as those involved often have different levels of status, knowledge and experience.

An action research study in a large international development organisation by Taut (2007) highlights the political nature of evaluation and the need to take the organisational work environment and the potentially negative effects of selfevaluation processes into account. Organisational and contextual challenges and issues that affected the outcomes of AC4SC included hierarchical structures that created issues with using PAR processes, regular turnover of M\&E coordinators, and loss of key leaders and other staff.

Employee turnover is a persistent challenge, especially in developing countries where there is often a shortage of people with evaluation skills, experience and capacities. This can undermine ECD efforts due to problems with maintaining capacity and skills and varying levels of commitment to the ECD process from new staff (Atkinson et al., 2005; Napp et al., 2002). In AC4SC, we found that the initial ability of EAN to roll out complex PM\&E systems was seriously constrained by a range of factors including loss of key M\&E staff, which created problems with continuity.

Our experiences indicate that it is both important to involve a wide range of staff in ECD activities, and to develop strategies to provide continuity of leadership and change 
agent roles, otherwise progress on ECD can be rapidly lost. As Horton et al. (2003: 55) suggest, this requires strategies such as setting up mechanisms that enable knowledge, skills and changed attitudes to be transferred to others within an organisation.

\section{A needs analysis for evaluation capacity development}

Our critical reflection on the experience of attempting to implement the AC4SC project and evaluation of the evidence produced through on-going meta-evaluation enabled us to identify a range of needs that must be met in order for ECD to succeed in development organisations in the long-term. These include, in no particular order of priority, the need for:

- A clear understanding of the context, including existing evaluation systems and capacities;

- A participatory implementation process;

- A combination of related approaches, including participatory evaluation, action research and creative approaches to organisational capacity development;

- Capacity building across the whole organisation, including stakeholders, especially community members; 
- A focus on the communicative and relational aspects of the process;

- Embedding evaluation into every part of the program development cycle;

- Development of a culture of learning within an organisation;

- Incorporation of local/indigenous knowledge, creativity and ideas;

- Building a wide range of skills, knowledge, attitudes, values and awareness;

- A critical approach to ECD that acknowledges challenges and issues;

- Ongoing meta-evaluation of ECD activities.

We now describe how these features of the holistic approach to ECD were identified and applied in the AC4SC project.

Understanding the context, including existing evaluation systems and capacities

An understanding of the local context and culture is important in ECD. A key starting point is understanding 'the history, structure, culture and context of the organisation' (Fitzpatrick, 2012: 237), as well as the context of the initiatives being evaluated and existing M\&E systems and capacities.

AC4SC built on earlier research that helped to provide some understanding of the organisation and its interest in taking a new approach to evaluation. Prior to AC4SC there was a lack of leadership in evaluation within EAN. There was no M\&E manager and only a small M\&E team. Feedback systems were poor and a more coordinated and 
rigorous approach to data management and analysis was needed. Indicators that were used to assess program effectiveness and impact were developed without community input and were often seen as unrealistic and not very useful. M\&E was mainly based on time-bound studies and 'success stories' derived from feedback that often came from letters sent by literate listeners who were unrepresentative of the listener population.

To more effectively plan and implement the AC4SC project, a detailed 'baseline' report was initially prepared by Michael Wilmore. This covered the history and structure of the organisation, the development of its M\&E systems, current M\&E activities and challenges, and various contextual challenges related to issues such as communication, travel, limited internet access and the large variety of linguistic groups in Nepal. Initial workshops with EAN staff included identifying challenges and issues in evaluating C4D initiatives and strategies for building M\&E into their everyday C4D activities, and a critical review their use of EAR. Analysis of questionnaires completed by 13 EAN staff at the start of AC4SC found that most had a moderate level of experience with PM\&E, a working knowledge of various M\&E methods, and wanted more skills in a wide range of M\&E methods. Lack of time to undertake M\&E was identified as a key issue. 


\section{Participatory implementation processes}

A key aim of this ECD approach is to contribute to the long-term process of encouraging continuous and active community and stakeholder participation and engagement in development initiatives and their evaluation. Lennie and Tacchi (2013:

3) argue that this process increases the long-term sustainability and effectiveness of C4D initiatives and that 'communication and this alternative approach to evaluation are critical for sustainable development'. In this approach, evaluation is seen as an ongoing, action learning, project development and improvement and capacity development process. The aim is that this process becomes embedded into an organisation's culture and its project planning and management processes, along with regular monitoring of and critical reflection on the evaluation process. However, participatory approaches to evaluation and ECD, such as those used in AC4SC, require greater planning and higher levels of participation and engagement than other evaluation approaches (Diaz-Puente et al., 2008).

The learning-based component of Lennie and Tacchi's framework for evaluating C4D is particularly relevant to this approach. This is based on action learning and PAR principles and processes that seek to achieve good communication, cooperation, collaboration and trust between those involved. The aim is to facilitate and encourage 
continuous learning, mutual understanding, empowerment, creative ideas and thinking, and responsiveness to new ideas and different attitudes, values and knowledge.

AC4SC was seen by some EAN staff who were interviewed two years after the project began as already helping EAN to look at M\&E in a different way and to improve the quality of their work. This included understanding the value of taking a flexible, inclusive, 'bottom-up' approach and continually adjusting and improving what they do. This new understanding is exemplified by the following comment from Naresh ${ }^{2}$, a program officer who actively participated in AC4SC:

We've learned the 'learning by doing' process. We would probably be more rigid if we were not to implement the AC4SC. We've learned there's always a place for adjustment and improvements within a project. It's not totally rigid. Equal Access, though we always valued our target audience, but we learned to do things from a bottom-up approach. We do not isolate people. We do not make decisions on their behalf. We try and include those people and make them feel that it's their project. It's trying to have this feeling of ownership. 


\section{Combining related approaches}

This approach draws on and combines a range of related methodologies and approaches, including action learning, action research, participatory evaluation, and holistic and creative approaches to organisational capacity development. It is underpinned by the learning organisation concept and the goal of building an evaluation culture in development organisations.

Participatory evaluation methodologies have close synergies with creative and holistic approaches to organisational capacity development such as those described by Pearson (2011). The strengths of these approaches include: they use a 'learning by doing' approach, enable rapid feedback about the success or failure of an ECD intervention, and can be cost-effective (Djamankulova et al., 2010; Forss et al., 2006; Taut, 2007). Findings from a recent synthesis of the ECD literature 'confirm the importance of participatory processes in ECB strategies' (Labin et al. 2012: 324). Participatory evaluation methodologies are particularly valuable and appropriate for ECD in complex settings where the context is 'impossible to manage' (Valery and Shakir, 2005: 87). A key benefit is that they can demystify the evaluation processes and make them more accessible to a wider range of stakeholders, including community members. 
AC4SC's participatory approach to research and evaluation was highly valued by EAN staff who were interviewed two years into the project. Several interviewees had received training in EAR and understood the value of this approach to understanding social change and local communication networks, systems and barriers. They reported valuing the AC4SC approach because it allowed EAN to understand things from a 'bottom-up' perspective, helped them to know their audiences better, understand 'the reality' of people's lives, and to provide a 'real picture' of a diversity of listener's views of their programs. They indicated that they had been unable to do these things effectively through their existing M\&E methods. This approach also helped them to identify the most useful tools to use in different contexts and with different groups.

Once EAN's M\&E staff had developed sufficient understanding and capacities in the AC4SC approach and key tools and methods, over time they trained 11 community researchers ${ }^{3}$ in five districts to use a wide range of participatory research tools and techniques, including the Most Significant Change (MSC) technique (Davies and Dart, 2005). This activity built on the established community reporter model that EAN already used. Community reporters were already undertaking EAR-type work when the project began. The selected CRs were young people who had good networks in the community, regularly listened to the radio programs being evaluated, and had the capacity to organise, undertake and report on participatory research activities on an 
ongoing basis. The work of the CR network was seen by several interviewees as an important means of documenting and observing the process of gradual change in a community and gathering what they referred to as 'in-depth', 'honest' and 'genuine' information.

Over time, use of the MSC technique in AC4SC proved useful for understanding a diversity of program impacts, including positive changes in gender and caste discrimination and political empowerment, and for engaging community members in discussion about social change issues. The MSC technique was used to complement other existing monitoring mechanisms based on letters, emails, SMS and feedback forms. An in-depth review in 2009 of the initial trial of MSC by the CRs found that while most stories contained at least some useful and interesting information about program impacts, they lacked detail about the changes experienced and did not use the MSC format (as set out in the Davies and Dart manual) very well. However, the review identified valuable examples of MSC stories that were later included in detailed MSC manuals which drew on learnings from the review. They clearly explained each step in the technique and included information and tips that were relevant to AC4SC and the collection of stories about the radio programs being evaluated. 


\section{Capacity building across the whole organisations and with stakeholders}

The active participation of a wide range of people in ECD is encouraged by the creative and flexible use of action learning-based processes that aim to build the capacity of whole organisations, along with their partners and stakeholders, including community members. This strategy can help to cushion the impact of staff turnover (Gibbs et al., 2009), a key problem in development organisations and an issue that affected AC4SC.

A key influence here was the approach to organisational capacity development detailed in Horton et al. (2003). They suggest that, rather than focussing on building the capacities of individuals and parts of an organisation, as in traditional approaches, it is more effective to focus on building the capacity of the organisation as a whole and to encourage the active participation of a broad range of staff and stakeholders in the process. This process is similar to the holistic approach to ECD that we advocate.

As far as we could, we used this approach in AC4SC, including adopting a partnership approach by encouraging EAN staff to share responsibility for facilitation of workshops and meetings, and organising meetings that engaged various stakeholders in the project. We also collaboratively developed manuals and other information made available via the project website. This helped the M\&E team to transfer their knowledge to the CRs, and to other staff of EAN and local development organisations. 
Our meta-evaluation of AC4SC indicated that internal training by the M\&E team conducted as part of AC4SC helped to build M\&E capacities more broadly in the organisation and to increase appreciation of the value and importance of evaluation to the ongoing program development and improvement process.

\section{Focussing on the communicative and relational aspects of the process}

As Horton et al. (2003: 56) point out, organisational capacity building is a process that 'evolves over a number of years [and] ... the development and maintenance of good working relationships between the various parties involved in a capacity development effort is crucial to its overall success'. This stresses the communicative and relational aspects of evaluation and, as in C4D, effective dialogue and interaction is a key feature of the process.

At the start of AC4SC we identified a need to develop more effective communication, collaboration and feedback systems within EAN and between EAN and its stakeholders. The M\&E team saw the program production team as resistant to changing existing M\&E systems and reluctant to take account of negative feedback on their programs. However, this changed over time after the M\&E and program production teams began 
meeting more regularly and $M \& E$ reports improved and became more useful to the program production teams, who started using this data to improve their programs.

An important outcome of AC4SC was that cooperation, communication, dialogue and interaction between the $M \& E$ team and the content production team improved over time, as reported though the regular meetings held between the EAN M\&E team and Australian team ${ }^{4}$, and through feedback provided through methods such as questionnaires and interviews. As a result, the content team gradually developed more trust and confidence in the M\&E team to provide 'reliable' data. In addition, workshops held in 2009 that brought the CRs and the content teams together for the first time helped them learn from each other and work better as a team.

\section{Embedding evaluation into every part of the program development cycle}

One of the most ambitious aims of ECD is the integration of evaluation into the whole program development and implementation cycle from the conception, design, and planning stages. It involves a diversity of staff, stakeholders and community members taking responsibility for research and evaluation activities. This process is seen as helping to develop the wide range of evaluation capacities that are required in this approach. Describing the realistic component of their framework for evaluating C4D, 
Lennie and Tacchi (2013: 36) suggests that this means that evaluation becomes 'a responsive and integral part of the iterative process of developing, implementing, improving and adjusting C4D initiatives'. They advise that this involves using an approach that is 'not rushed, and allowing dialogue to begin the process' and that in consultation with a range of participants, the process would also include 'developing flexible and realistic plans and timeframes for the whole evaluation process, using an organic approach that is responsive to unfolding developments' (Lennie and Tacchi, 2013: 36).

The AC4SC methodology aimed to facilitate the participation of EAN staff and primary stakeholders in all aspects of the evaluation process and involved careful planning of M\&E work and ongoing adjustment of these plans as program objectives changed. It included the use of participatory and mixed methods research and evaluation tools and techniques, and triangulation of data to increase rigour. The basic processes of this methodology included 'listening to audiences, learning from this knowledge, systematically processing it and feeding it back into the organization and its practices in an ongoing cycle' (Lennie and Tacchi, 2013: 40-41). At the 2013 follow up meeting, EAN staff reported that their radio programs would not succeed if there was no M\&E mechanism to provide regular feedback and that this had helped their programs 'grow even further and stronger'. 


\section{Developing a culture of learning within an organisation}

Learning organisations regularly critically reflect on their systems, processes, internal and external relationships and feedback systems, and identify ways they can be improved. These processes are important to more effective and sustainable development practices. Behrens and Kelly (2008: 44) point out that 'in a learning organisation paradigm, evaluation becomes part of the change effort'. Labin et al. (2012: 307) note that ECD has attracted the interest of evaluators 'committed to increasing stakeholder understanding of evaluation and building evaluation culture and practice in organizations'. Preskill and Boyle (2008: 453) suggest that if organisations support and encourage 'organizational learning capacity' it is more likely that ECD activities will be successful. The support of managers and leaders is vital to the development of learning organisations and they need to be seen as strong models for learning (Forss et al., 2006; Hoole and Patterson, 2008; Taut, 2007).

In AC4SC we found it important to obtain the support of all management staff in EAN, to engage them in key activities whenever possible, and to maintain regular communication with them about progress with the project. Analysis of interviews with EAN staff found that the concept of a learning organisation and its associated practices was seen as particularly important to Equal Access and other C4D organisations. 
Senior management staff member Ramesh indicated his strong support for this goal when he stated: As a communication organisation, we have to be a learning organisation, that's even more important. EAN interviewees identified a wide range of features of a learning organisation which are similar to those in the literature (Ortenblad, 2013; Raeside, 2011; Pearson, 2011), including being open to feedback from a range of sources, being open to talking about 'weaknesses' and 'mistakes', increasing the appreciation of M\&E across the organisation, and developing and adjusting systems, processes and knowledge that helps them to continuously improve. Ramesh saw the more openly critical approach that EAN took as something that other organisations did not do because of the pressure to 'satisfy the donor'. Devraj, a content production manager, also commented on the need to encourage staff to be 'critical' and 'analytical' as part of the learning process. Some staff who were interviewed in 2009 thought that EAN was already moving towards becoming a learning organisation. They thought this was indicated by M\&E being considered as an integral part of the organisation and having staff who understood its value and were actively engaged in research and M\&E. In addition, some content production staff indicated that they now understood the 'learning by doing', continuous improvement approach of AC4SC. Participation in AC4SC was seen as an important factor in these changes. Two M\&E team members thought the project had been effective in building a 
research culture within EAN while other interviewees reported that the M\&E team regularly shared its knowledge and research findings with other staff.

Incorporating local/indigenous knowledge, creativity and ideas

Another important feature of this approach is that it seeks to tap into and incorporate local/indigenous knowledge, creativity and ideas, and social change aspirations and needs into ECD efforts. It also aims to empower local staff and communities so that their knowledge, ideas and learnings can be effectively utilised and acted upon (Hay, 2010; Raeside, 2011).

The development of flexible, community-based research and evaluation approaches and methodologies and the long history of the use of PAR in the development field highlights the need to look to local knowledge, ideas and innovation in order to develop appropriate, effective and innovative evaluation approaches and methods. Hay (2010: 229) proposes that 'Instead of looking to the north for curriculum and methods, [evaluation] field building entails experimentation and indigenous innovation, building on the best ideas available but creating something better'. 
Carden (2007: 53) makes the case for development evaluation being 'best done by locally based researchers and organizations who know the culture and context ... and have a responsibility to build capacity to use research in decision-making in local institutions, governmental, corporate or non-governmental'. This indicates a need to better appreciate locally developed learning and evaluation methods and more appropriate and effective evaluation methodologies that have been developed and tested with people in the development context. Over four years we worked closely with EAN to collaboratively develop, test and refine the methodology and each component. This resulted in the production of the Equal Access Participatory Monitoring and Evaluation Toolkit (Lennie et al., 2011), based on the experiences, learnings and critical reflections of EAN staff.

The Equal Access toolkit includes a module on 'Critical Listening and Feedback Sessions' (CLFS), which was initiated and developed by Bikash Koirala with input from others involved in AC4SC. The idea of each session is to randomly choose any episode of a broadcasted radio program and ask all EAN staff to listen critically and participate in a feedback session. This process has encouraged program team members to consider their radio programs more critically and to continually make improvements. Bikash recently advised that 'CLFS has been incorporated into most of EAN's projects to monitor radio programs at the centre and community levels. It has helped us to get 
critical insights instantly and has also built the capacity of our FM radio station partners to monitor their radio programs. So it's like knowledge transfer'.

In addition, EAN has increasingly incorporated the use of new ICTs in its M\&E practices, including drawing on SMS and Interactive Voice Recorder messages from listeners and using smart phones to report on case studies.

\section{Building a wide range of skills, knowledge, attitudes, values and awareness}

A wide range of skills, knowledge, attitudes, values and awareness are required for the effective evaluation of development programs and initiatives. Effectively using participatory and systems-oriented evaluation approaches and facilitating better use of evaluations requires 'people skills' (Patton, 2010: 49), including skills in relationship building and interpersonal communication. A wide range of skills are needed to successfully undertake participatory research and evaluation (Hearn et al., 2009; Taut, 2007). As well as technical skills, they include: 'strong skills in facilitation, as well as humility, respect for others and the ability to listen' (Narayan, 1993, cited in Boyle, 1999: 143). Other skills include: 'responsiveness to user needs ... acceptance of diverse

views, [and the] ability to establish rapport and trust' (Green, 1988, cited in Taut, 2007: 
49). High level conflict management and facilitation skills are also needed when stakeholders have contradictory perspectives or unequal power.

Capacity building activities conducted during AC4SC aimed to develop a wide range of facilitation, communication and people skills. For example, workshops with a variety of EAN staff held in September 2007 included small groups practicing powerful listening and other communication-related skills. Analysis of feedback questionnaires found that key outcomes from these activities included improved 'team building', 'team spirit' and communication, and appreciation of the need for a 'culture of sharing' among EAN team members. In addition, during follow up research in 2012, some of the CRs commented on the need to learn how to build trust, noting that this was an important skill.

\section{A critical approach to ECD that acknowledges challenges and issues}

This approach takes a critical perspective that acknowledges the many challenges, tensions and issues that can hinder the effectiveness and sustainability of ECD in the development context. Those identified by Lennie and Tacchi (2013: 100-104) include:

- Contextual factors in poor, politically unstable, developing countries 
- Power relations in ECD projects

- The complexity of evaluating C4D

- Attitudes to evaluation among donors, C4D organisations and NGOs

- Maintaining and sustaining evaluation capacity

- Facilitating wide participation in evaluation of C4D

- The wide range of skills required in evaluating C4D.

At regular points in AC4SC, EAN staff were encouraged to critically reflect on the project and provide feedback on what was working well and less well, and the impacts of the project on themselves and EAN. Most of the staff provided positive feedback on the initial capacity building activities. However, in a later review of AC4SC by an Equal Access International manager, feedback was received that the level of complexity of the methods and impact assessment framework was leading to confusion and few staff could clearly articulate the project's aims and objectives. There was a preference for a methodology that was 'much simpler and practical'. An M\&E team member commented: 'From the start, everything is new, new methods, new feedback, new ideas. ... we decide a certain thing and the academics will come with another issue or idea or thing we need to do'. What they needed was 'more practical guidance to show us the bridge between the academic and the practical' (Tacchi et al., 2013: 153). This highlights a key challenge for projects such as AC4SC which have both academic and 
practical aims: 'the need to balance these aims in ways that reduce confusion and feelings of being overwhelmed by too many new ideas and methods at once' (Tacchi et al., 2013: 153). This may require spending more time in the initial planning phase on ensuring that the ECD objectives and process is clear to everyone involved and not too ambitions or unrealistic in its scope. The roles and responsibilities of everyone involved also need to be very clear.

\section{Ongoing meta-evaluation of ECD activities}

Recent studies suggest that meta-evaluation can be valuable in developing new approaches to evaluation, building evaluation capacities, and enhancing organisational learning (Hanssen et al., 2008; Uusikyla and Virtanen, 2000). An important component of the holistic ECD approach is using an ongoing meta-evaluation process to continually improve ECD activities and increase their sustainability and success.

Our ongoing, rigorous meta-evaluation of AC4SC assessed the effectiveness of this approach, including for building an evaluation culture and improved $M \& E$, communication and feedback systems within EAN as a whole. Key objectives of the meta-evaluation included: continuous development, adaptation and improvement of the impact assessment methodology, M\&E systems and process and other project 
activities; identification of project impacts ; and capacity building in critical reflection and review. This meta-evaluation was essential to understanding various constraints related to the organisational context that affected the success of the project and the development of improved M\&E systems and capacities within EAN (Lennie et al., 2012).

\section{Strategies for an effective and sustainable holistic, learning-based approach to evaluation capacity development}

We have identified a number of principles and strategies for an effective and sustainable holistic, learning-centred approach to ECD at the organisational and community levels:

- Assess an organisations' readiness for learning and change and its existing M\&E capacities, systems and processes.

- Understand the organisational culture, dynamics and wider context; take this into account in the design and implementation of ECD.

- Draw on local innovation and experimentation in the ECD process and encourage self-organisation and creative risk-taking. 
- Foster a learning organisation and an evaluation culture through the support and influence of leaders and managers.

- Embed evaluation into the whole program development cycle.

- Design ECD activities that are flexible and open to continuous adaptation and revision.

- Pay attention to the communicative and relational dimensions of ECD, and develop relationships based on mutual trust, knowledge sharing, open communication and feedback systems.

- Empower local staff and communities to share and act on the knowledge and understandings gained from evaluation to better utilise the results of participatory evaluation and research.

- Engage in regular critical reflection of ECD activities to continually improve them.

As we have indicated, the holistic ECD approach takes more time and resources than standard training-oriented approaches. The funding, resources and support required for long-term ECD can be difficult for development organisations to find. This problem was particularly acute for AC4SC and EAN; the Global Financial Crisis (GFC) that swept the world in 2008 occurred during the project and continued to have very significant impacts on the ability of the organisation to sustain its activities. Throughout the latter half of the project there was constant pressure to do more with less. However, on 
reflection we consider this to have been largely beneficial, because these significant financial constraints forced everyone involved to consider how the holistic ECD approach could be made sustainable following the end of the project's four-year funding.

EAN staff who were interviewed in 2009 were therefore asked to suggest ideas about how to make their new M\&E systems and the AC4SC approach more sustainable. They put forward a range of useful ideas and strategies, including:

- Embedding and institutionalising the whole AC4SC process into EAN by building this in from the start of planning programs. This requires involving stakeholders more in the process, continuing to build capacity in M\&E, and valuing data from field staff.

- Including details of the AC4SC methodology in proposals to donors to help them obtain ongoing funding, given that this methodology was seen as something unique, that gave EAN a 'competitive advantage'. EAN has continued to refer to the toolkit developed during AC4SC in almost every funding application.

- Expanding the CR network and the M\&E team and creating more ways to make the CR network useful, such as using CRs as a resource for partner organisations once they reached a higher skill level. 
- Offering training in the AC4SC process to other organisations and turning EAN into a 'resource centre' for PM\&E in Kathmandu.

Since the AC4SC project ended EAN has been regularly asked to provide training in the MSC technique by UNICEF and other development organisations in Kathmandu. This has helped M\&E staff to maintain key evaluation capacities developed during AC4SC and to share their expertise with others. During 2012 EAN faced serious problems with obtaining funding for its projects, resulting in a loss of key M\&E staff and the disbanding of the CR network. However, by late 2012 EAN had successfully obtained funding for a major five-year radio project, which is currently being implemented by PACT. This project has its own separate M\&E section and currently employs 17 community action researchers (CARs), as they are now called, in six districts in Nepal. The CR manual in the AC4SC toolkit was used to train the new CARs. A former CR helped to conduct this training and now works for the PACT project. Donors are also providing EAN with more funding for M\&E. 


\section{Conclusion}

Preskill and Boyle (2008: 457) believe that ECD 'represents the next evolution of the evaluation profession and, as such, has the potential for transforming the field in ways only imagined'. However, in order to realise this transformative potential it is vital that we recognise potential barriers to successful ECD and build effective counter-measures into implementation strategies from the outset. A flexible, emergent approach is required that is open to change and continuous adjustment based on regular feedback and critique from those involved. The complexity of the challenges faced in the AC4SC project and its length (four years with subsequent post-project evaluations over the following two years) provided an unprecedented opportunity to explore these barriers and devise solutions that were tested in practice and subjected to rigorous metaevaluation. Drawing on this evidence, we have identified a number of principles and requirements for the successful implementation of a holistic, learning-centred approach to ECD. These are essential if the transformative potential of this approach is to be realised by development organisations that may not have the luxury of experimenting with this approach due to severe funding and resource constraints.

The most important conclusion we draw from this experience is that ECD cannot be implemented as a separate 'tool' or 'technique', but must be founded on wider and 
more fundamental changes to organisational culture. In particular, the absence within an organisation of openness to learning, especially learning founded on the critical analysis of norms, communication systems and relationships between those involved, can undermine the development of both holistic perspectives on problems that ECD is intended to overcome and the effective participation of all stakeholders. The latter is a key requirement for implementing any practical actions as an outcome of the evaluation process. We suggest that the inclusion of rigorous meta-evaluative activity is crucial to ECD. This is because it enables organisations to identify when and how the absence of these foundational capacities for learning and critical self-evaluation may, ironically prevent them from using evaluation as a means to continuously improve development programs and increase their impact in ways that better meet community needs. If these barriers and issues can be overcome, our research suggests that the holistic, learning-based approach to ECD, combined with participatory forms of development and evaluation, can make an important contribution to increasing the sustainability of development organisations and, in the long run, to more effective and sustainable development. 


\section{Acknowledgements}

We would like to thank and acknowledge everyone who participated in the AC4SC project and provided feedback and input into it. We also acknowledge the contributions of Andrew Skuse from the University of Adelaide.

\section{Funding}

The AC4SC project was funded by the Australian Research Council (grant number LP0775252), USAID/Nepal and Equal Access.

\section{Notes}

1. We use the term 'evaluation capacity development' (ECD) since this term is most often used in the field of development evaluation, however 'evaluation capacity building $(E C B)$ is also used in much of the literature we draw upon in this paper. While the literature often refers to these terms interchangeably, Carter (2013) notes that some experts distinguish between them. In this paper we use ECD even when referring to work that uses the term ECB, except when we are directly quoting, in which case ECB is used.

2. All names of EAN staff who were interviewed have been changed. 
3. Eleven CRs were trained but only eight of them provided significant amounts of data.

4. We met regularly through phone and/or Skype meetings, as well as less regular face to face meetings in Nepal. The regularity of phone/Skype meetings varied across the project timespan, from quarterly, to bi-weekly, depending on project needs and activities.

\section{References}

Armytage, L (2011) Evaluating aid: an adolescent domain of practice. Evaluation 17(3): 261-276.

Atkinson, D, Wilson, M and Avula, D (2005) A participatory approach to building capacity of treatment programs to engage in evaluation. Evaluation and Program Planning 28: 329-334.

Ba Tall, O (2009) The role of national, regional and international evaluation organisations in strengthening country-led monitoring and evaluation systems, In: Segone M (ed) Country-led Monitoring and Evaluation Systems. Better Evidence, Better Policies, Better Development Results. Geneva: UNICEF, pp.119-134. 
Bayley S, Ben Younes Chaouch S, Bilinsky P, Brown A, Crail J, Hsu L-N et al. (2012) An international workshop agreement on evaluation capacity development, 17-21 October 2011, Evaluation Capacity Development Group, Geneva, Switzerland. URL (consulted 10 September 2014): http://www.ecdg.net/wpcontent/uploads/2012/03/IWA-on-ECD6.pdf

Behrens T and Kelly T (2008) Paying the piper: foundation evaluation capacity calls the tune. In: Carman J and Fredericks K (eds), Nonprofits and evaluation. New Directions for Evaluation 119: 37-50.

Boyle R (1999) Professionalising the evaluation function: human resource development and the building of evaluation capacity. In: Boyle R and Lemaire D (eds) Building Effective Evaluation Capacity. Lessons From Practice. New Brunswick: Transaction Publishers, pp.135-151.

Carden F (2007) The real evaluation gap. Alliance 12(4): 53-54.

Carter B (2013) Evaluation capacity development. Helpdesk Research Report, GSDRC, 17 September. URL (consulted 10 September 2014):

http://www.gsdrc.org/docs/open/HDQ996.pdf 
Cousins JB, Whitmore E and Shulha L (2013) Arguments for a common set of principles for collaborative inquiry in evaluation. American Journal of Evaluation 34(1): 7-22.

Cracknell BE (2000) Evaluating Development Aid: Issues, Problems and Solutions. Thousand Oaks: Sage.

Davies R and Dart J (2005) The 'Most Significant Change' (MSC) Technique. A Guide to its Use. (consulted 10 September 2014):

http://www.mande.co.uk/docs/MSCGuide.pdf

Dabelstein N (2003) Evaluation capacity development: lessons learned. Evaluation 9(3): 365-369.

Djamankulova K, Temirova N and Sobirdjonova M (2010) Using action learning sets methodology in an NGO capacity building programme. Praxis Note No. 53, INTRAC. Fitzpatrick JL, Sanders JR and Worthen BR (2012) Program Evaluation: Alternative Approaches and Practical Guidelines, 4th ed. New Jersey: Pearson.

Forss K, Kruse S, Taut S and Tenden E (2006) Chasing a ghost? An essay on participatory evaluation and capacity development. Evaluation 12 (1): 128-144. 
Gibbs DA, Hawkins SR, Clinton-Sherrod AM and Noonan RK (2009) Empowering programs with evaluation technical assistance. Outcomes and lessons learned. Health Promotion Practice 10(1): 385-445.

Hanssen C, Lawrenz F and Dunet D (2008) Concurrent meta-evaluation. A critique. American Journal of Evaluation 29(4): 572-582.

Hay K (2010) Evaluation field building in South Asia: reflections, anecdotes, and questions. American Journal of Evaluation 31(2): 222-231.

Hearn G, Tacchi J, Foth M and Lennie J (2009) Action Research and New Media: Concepts, Methods and Cases. Cresskill, NJ: Hampton Press.

Hoole E and Patterson T (2008) Voices from the field: evaluation as part of a learning culture. In: Carman J and Fredricks K (eds) Nonprofits and Evaluation. New Directions for Evaluation 119: 93-113.

Horton D, Alexaki A, Bennett-Lartey S, Brice KN, Campilan D, Carden F et al. (2003) Evaluating Capacity Development: Experiences from Research and Development Organizations Around the World. The Hague: International Service for National Agricultural Research. 
Kuzmin A (2010) Use of evaluation training in evaluation capacity building. In: Segone M (ed) From Policy to Results. Developing Capacities for Country Monitoring and Evaluation Systems. New York: UNICEF, pp.240-251.

Labin S, Duffy J, Meyers D, Wandersman A and Lesesene C (2012) A research synthesis of the evaluation capacity building literature. American Journal of Evaluation 33(3): 307-338.

Lennie J and Tacchi J (2011) Researching, monitoring and evaluating communication for development: Trends, challenges and approaches, Report on a literature review and consultations with Expert Reference Group and UN Focal Points on C4D. Prepared for the United Nations Inter-agency Group on Communication for Development, New York: UNICEF URL (consulted 10 September 2014): http://www.unicef.org/cbsc/files/RME-RPEvaluating_C4D_Trends_Challenges_Approaches_Final-2011.pdf Lennie, J. and Tacchi, J. (2013) Evaluating Communication for Development: A Framework for Social Change. Abingdon: Routledge.

Lennie J and Tacchi J (2014) Bridging the divide between upward accountability and learning-based approaches to development evaluation. Strategies for an enabling environment. Evaluation Journal of Australasia, 14(1): 12-23. 
Lennie J, Tacchi J, Koirala B, Wilmore M and Skuse A (2011) Equal Access Participatory Monitoring and Evaluation Toolkit, Queensland University of Technology, University of Adelaide, and Equal Access Nepal. URL (consulted 10 September 2014):

http://betterevaluation.org/toolkits/equal_access_participatory_monitoring.

Lennie J, Tacchi J and Wilmore M (2012) Meta-evaluation to improve learning, evaluation capacity development and sustainability: findings from a participatory evaluation project in Nepal. South Asian Journal of Evaluation in Practice 1(1): 13-28.

Morgan P (2013) Evaluating capacity development. In: Donaldson S, Azzam T and Conner R (eds) Emerging Practices in International Development Evaluation. Charlotte, NC: Information Age Publishing, pp.75-104.

Naccarella L, Pirkis J, Kohn F, Morley, B, Burgess P and Blashki G (2007) Building evaluation capacity: definitional and practical implications from an Australian case study. Evaluation and Program Planning 30(3): 231-236.

Napp D, Gibbs D, Jolly D, Westover B and Uhl G (2002) Evaluation barriers and facilitators among community-based HIV prevention programs. AIDS Education and Prevention 14 Supp. A: 38-48. 
Ofir Z and Kumar AK (2013) Evaluation in developing countries. What makes it different?' In: Donaldson S, Azzam T and Conner R (eds), Emerging Practices in International Development Evaluation. Information Age Publishing, Charlotte, NC, pp. 11-24.

Ortenblad A (2013) What do we mean by ‘learning organization'? In: Ortenblad A (ed) Handbook of Research on the Learning Organization: Adaptation and Context. Cheltenham: Edward Elgar, pp.22-34.

Patton MQ (2010) Future trends in evaluation. In: Segone M (ed) From Policy to Results. Developing Capacities for Country Monitoring and Evaluation Systems. New York: UNICEF, pp.44-57.

Patton, MQ (2011), Developmental Evaluation: Applying Complexity Concepts to Enhance Innovation and Use. Guilford Press: New York.

Pearson J (2011) Creative Capacity Development: Learning to Adapt in Development Practice. Sterling VA: Kumarian Press.

Preskill, H (2010) Exploring effective strategies for facilitating evaluation capacity building. In: Segone M (ed) From Policy to Results. Developing Capacities for Country Monitoring and Evaluation Systems. New York: UNICEF, pp.224-238. 
Preskill H and Boyle S (2008) Multidisciplinary model of evaluation capacity building. American Journal of Evaluation 29 (4): 443-459.

Raeside A (2011) Are INGOs brave enough to become learning organisations? In: Ashley $\mathrm{H}$, Kenton $\mathrm{N}$ and Milligan $\mathrm{N}$ (eds.) How Wide are the Ripples? From Local Participation to International Organisational Learning, Participatory Learning and Action 63: 97-102. London: The International Institute for Environment and Development.

Robinson T and Cousins B (2004) Internal participatory evaluation as an organizational learning system: a longitudinal case study. Studies in Educational Evaluation 30: 1-22.

Schiavo-Campo S (2005) Building country capacity for monitoring and evaluation in the public sector: Selected lessons of international experience, Evaluation Capacity Development, ECD Working Paper Series No. 13: The World Bank Operations Evaluation Department. URL (consulted 10 September 2014): http://preval.org/files/2086.pdf

Segone M (ed) (2009) Country-led Monitoring and Evaluation Systems. Better Evidence, Better Policies, Better Development Results. Geneva: UNICEF. 
Stern E, Stame, N, Mayne, J, Forss, K, Davies, R and Befani, B (2012) Broadening the range of designs and methods for impact evaluation. DFID Working Paper 38. London: DFID.

Sonnichsen, R (1999) Building evaluation capacity within organisations. In: Boyle R and Lemaire D (eds) Building Effective Evaluation Capacity. Lessons from Practice. New Brunswick and London: Transaction Publishers, pp.53 -73.

Tacchi, J (forthcoming) Ethnographic action research: project development and evaluation. In: Bradbury-Huang, $\mathrm{H}$ (ed.) Handbook of Action Research: Participative Inquiry and Practice. Oxford: Sage.

Tacchi J and Lennie J (2014) A participatory framework for researching and evaluating communication for development and social change. In: Wilkins KG, Tufte T and Obregon R (eds) The Handbook on Development Communication and Social Change. Oxford: Wiley Blackwell, pp. 298-320.

Tacchi J, Lennie J and Wilmore M (2013) Critical reflections on the use of participatory methodologies to build evaluation capacities in international development organisations. In: Goff S (ed) From Theory To Practice; Context in Praxis. Selected Papers from the $8^{\text {th }}$ Action Learning, Action Research World Congress Australia 2010, Action Learning Action Research Association, Toowong, Queensland, pp. 150-160. 
Tacchi J, Fildes J, Martin K, Mulenahalli K, Baulch E and Skuse A (2007) Ethnographic Action Research Training Handbook. URL (consulted 10 September 2014) http://ear.findingavoice.org/

Taut S (2007) Studying self-evaluation capacity building in a large international development organization. American Journal of Evaluation 28(1): 45-59.

Uusikyla P and Virtanen P (2000) Meta-evaluation as a tool for learning. A case study of the European Structural Fund evaluations in Finland. Evaluation 6 (1): 50-65.

Valery R and Shakir S (2005) Evaluation capacity building and humanitarian organization. Journal of Multidisciplinary Evaluation 2(3): 78-112. 